\title{
Employeurs et anti-syndicalisme au Canada
}

Une étude juridique des stratégies mobilisées

Employers and Union Busting in Canada. A Legal Study

\section{Mélanie Laroche et Marie-Ève Bernier}

\section{(2) OpenEdition \\ Journals}

Édition électronique

URL : http://journals.openedition.org/travailemploi/6998

DOI : 10.4000/travailemploi.6998

ISSN : 1775-416X

Éditeur

DARES - Ministère du Travail

Édition imprimée

Date de publication : 1 avril 2016

Pagination : 51-74

ISSN : 0224-4365

Référence électronique

Mélanie Laroche et Marie-Ève Bernier, "Employeurs et anti-syndicalisme au Canada », Travail et Emploi [En ligne], 146 | avril-juin 2016, mis en ligne le 11 juillet 2019, consulté le 05 septembre 2019. URL:

http://journals.openedition.org/travailemploi/6998; DOI : 10.4000/travailemploi.6998 


\title{
Employeurs et anti-syndicalisme au Canada Une étude juridique des stratégies mobilisées
}

\author{
Mélanie Laroche ${ }^{*}$,Marie-Ève Bernier ${ }^{* *}$
}

\begin{abstract}
Cet article traite de l'anti-syndicalisme patronal et propose une analyse de la jurisprudence issue des cas entendus par les commissions des relations du travail de deux provinces canadiennes, le Québec et l'Ontario. Plus spécifiquement, notre analyse porte sur 145 décisions dans lesquelles il a été reconnu par les tribunaux compétents que les employeurs avaient eu recours à une ou plusieurs stratégies anti-syndicales ayant des effets discriminatoires. Nos résultats mettent en évidence la large palette des tactiques utilisées par les employeurs pour combattre la syndicalisation au travail, et ce, lors des différentes étapes de la vie d'un syndicat au sein d'une entreprise. Ils montrent une nette préférence pour les stratégies les plus agressives, celles qui visent la suppression pure et simple des syndicats. Ils attestent enfin que les pratiques de discrimination syndicale ciblent le plus souvent un seul salarié, mais peuvent aussi parfois viser un groupe, voire l'ensemble des salariés.
\end{abstract}

$\mathrm{L}$ 'érosion graduelle des droits syndicaux en Amérique du Nord a fait l'objet d'une abondante littérature (SuPIOT, 1999 ; TuRnER et al., 2001 ; AIDT, TZANNATOS, 2008 ; FAirbrother, YATES, 2003 ; EdWARds, 2004 ; KumAr, Schenk, 2005 ; Hickey et al., 2010). Parmi les défis auxquels doivent faire face les organisations de salariés figure l'anti-syndicalisme patronal. La littérature fait effectivement état d'une utilisation accrue des stratégies anti-syndicales par les employeurs (BRONFENBRENNER, 2009) et d'un renforcement d'une idéologie gestionnaire qui refuse le partage du pouvoir avec une tierce partie représentant les travailleurs (DuNDON et al., 2006 et 2010).

Pour que cette idéologie s'enracine dans les organisations de travail et afin de conserver un environnement de travail sans présence syndicale, des multinationales comme Walmart, McDonald's ou Disney ont adopté des positions claires contre la

\footnotetext{
* École de relations industrielles, Université de Montréal ; melanie.laroche.2@umontreal.ca.

** Faculté de droit, Université de Montréal ; marie-eve.bernier.1@ umontreal.ca.
} 
syndicalisation (DunDon et al., 2006), positions susceptibles d'influencer d'autres entreprises de leurs secteurs respectifs.

Contrairement à la situation américaine, la question de l'anti-syndicalisme patronal n'a pas fait l'objet de nombreuses études au Canada. Ce vide dans la littérature s'expliquerait par une acceptation plus grande du fait syndical par les employeurs canadiens, en particulier en raison d'une présence historiquement plus forte du syndicalisme dans le système des relations industrielles, mais aussi de lois qui encadrent davantage les droits d'association et de négociation collective des travailleurs (THOMASON, POZZEBON, 1998). Les quelques recherches réalisées ont pourtant montré une volonté clairement affirmée des employeurs canadiens de s'opposer à la négociation (cf. par exemple MarTinello, YATES, 2004).

Plus généralement, les travaux sur la question ont mis en évidence deux grands types de stratégies anti-syndicales : les stratégies dites de substitution et de suppression. Dans le premier cas, l'employeur offre aux salariés une voie alternative à la syndicalisation soit en augmentant leurs salaires et avantages sociaux, soit en favorisant leur consultation et implication dans l'entreprise (LAROCHE et al., 2015) : les syndicats ne sont pas directement attaqués, mais ils sont mis en concurrence avec des modes de gestion directs des aspirations des salariés, qui entendent se substituer à eux. Dans le second cas, il s'agit d'imposer des coûts (symboliques ou matériels) aux travailleurs afin de décourager leur adhésion à un syndicat, ou de les punir d'avoir adhéré (l'objectif à terme est alors la suppression du syndicat). Selon Kate BRONFENBRENNER (2009), les tactiques utilisées par les employeurs seraient de plus en plus de nature suppressive, incluant le licenciement (pour faute) ${ }^{1}$ et intimidations, menaces de fermeture et fermetures réelles d'établissement.

Les stratégies anti-syndicales peuvent être mobilisées à différentes étapes de la syndicalisation : avant la reconnaissance du syndicat comme partie à même de négocier pour les salariés (BENTHAM, 2002 ; BRONFENBRENNER, 1996, 2009 ; JURAVICH, BRONFENBRENNER, 1998 ; FREEMAN, KLEINER, 1990 ; LAROCHE et al., 2015 ; RIDDELL, 2001), au moment de négocier le premier accord collectif (BENTHAM, 2002 ; BRONFENBRENNER, 2009) ou même après (LAROCHE et al., 2015). Elles contribuent ainsi à diminuer les chances qu'un collectif soit représenté par un syndicat.

Que les stratégies soient substitutives ou suppressives, qu'elles soient déployées lors de la phase de syndicalisation ou une fois le syndicat reconnu, elles constituent un frein à l'organisation collective des travailleurs. Certaines d'entre elles ont également un effet discriminatoire : elles impliquent un traitement spécifique qui a pour but de désavantager certains individus parce qu'ils appartiennent à une organisation syndicale (CHAPPE, 2013). Compte tenu de ces conséquences possiblement négatives, il convient de bien comprendre les multiples formes que peuvent prendre les stratégies

1. C'est l'équivalent du « congédiement » au Québec, qui s'y distingue du « licenciement » (ce dernier correspondant au seul licenciement pour motif économique en France). Dans la suite du texte, le terme de licenciement sera utilisé pour désigner la notion de congédiement. 
anti-syndicales mises en œuvre par les employeurs. Cet article s'intéresse précisément à cette question, en analysant un corpus de décisions de justice rendues dans deux provinces canadiennes, le Québec et l'Ontario.

Pour ce faire, il se divise en quatre parties. La première présente les définitions adoptées pour ces stratégies et pratiques de discrimination anti-syndicales ainsi qu' une typologie les classant selon leur nature et le moment de leur mise en œuvre par les employeurs. L'état du droit canadien sur ces questions est ensuite présenté, avant que les considérations méthodologiques ne soient discutées. Enfin, la quatrième partie propose une analyse approfondie du contenu de la jurisprudence à partir du corpus de décisions retenues, afin de comprendre la nature et les multiples formes des stratégies patronales, fournissant des exemples précis et variés de pratiques de discrimination syndicale.

\section{Stratégies patronales anti-syndicales et pratiques discriminatoires : définitions et formes}

\section{Définitions}

Notre conception des stratégies anti-syndicales et des pratiques de discrimination syndicale nous amène à prendre en compte non seulement leur finalité (la substitution ou la suppression des syndicats) mais également leurs objectifs intermédiaires (empêcher la reconnaissance du syndicat ou nuire à son maintien comme partie à même de négocier pour les salariés). Notre cadre d'analyse considère les stratégies antisyndicales comme le reflet du degré d'opposition de l'employeur à l'acteur syndical (degré d'acceptation ou d'opposition) et du but général visé par celles-ci (substitution ou suppression). Afin de mieux cerner les intentions précises qui guident les actions de l'employeur, nous examinons les types de campagnes ${ }^{2}$ qu'il est susceptible de mener. La typologie que nous avons préalablement élaborée (LAROCHE et al., 2015), et qui sera présentée dans la prochaine section, distingue ainsi quatre types de campagne, dites de «cooptation », de «contrôle », de «peur » et de «blocage ». Selon le type choisi, l'employeur recourra à des moyens concrets, ou tactiques, pour atteindre ses objectifs. Il pourra par exemple distribuer des documents anti-syndicaux, licencier des syndicalistes, créer des comités anti-syndicaux, etc.

\footnotetext{
2. Au Canada, on parle de « campagne », par analogie aux campagnes politiques existant en France, dans la mesure où la présence syndicale dépend de l'appui manifesté par les membres de l'unité d'accréditation concernée (cf. infra, tableau p. 58 pour une définition de l'unité d'accréditation). D'une part, on appelle « campagne syndicale » la période pendant laquelle un syndicat tente d'obtenir suffisamment de voix pour être accrédité, soit par la signature d'un nombre suffisant de cartes d'adhésion, soit par la victoire à l'élection suivant la campagne. Le syndicat est alors reconnu comme le seul interlocuteur à même de négocier au nom des salariés avec l'employeur. D'autre part, on qualifie de « campagne anti-syndicale » la période pendant laquelle l'employeur utilise différentes méthodes pour empêcher l'implantation d'un syndicat au sein de son entreprise.
} 
Plusieurs de ces tactiques ont un effet discriminatoire. Alors que la notion de « discrimination » fait généralement référence à des «traitements inégalitaires ou différenciés en fonction du sexe ou de la couleur de peau », la discrimination syndicale désigne plus particulièrement les « différences de traitement entre salariés "comparables" - différences en termes de rémunération, d'évolution de carrière, de reconnaissance entre militants syndicaux et salariés de même profil (même qualification, ancienneté, voire compétence) » (DEBRÉGEAS, 2013,p. 26 ; CHAPPE, 2015). Autrement dit, certains individus vont recevoir, en raison de leur appartenance syndicale, un traitement désavantageux (CHAPPE, 2013). La discrimination sera en ce sens considérée comme syndicale lorsqu'elle se fonde sur trois critères illégaux de distinction entre les salariés : l'appartenance syndicale, qui vise un salarié qui adhère à un syndicat ; l'activité syndicale d'un délégué ou d'un représentant ; enfin, le mandat représentatif d'un militant qui assume des fonctions de représentation non strictement syndicale, par exemple, les participants à un comité d'amélioration continue ou de santé et sécurité au Québec, et le délégué du personnel ou le conseiller prud'homme en France (SPIRE, 2006). Les militants syndicaux, fréquemment exposés à des risques accrus de conflits avec leur employeur, sont ainsi plus souvent contraints de faire des sacrifices de carrière (BÉROud et al., 2008). En outre, ces pratiques contribuent à décourager les autres travailleurs de s'engager syndicalement (SPIRE, 2006).

Les pratiques discriminatoires prennent plusieurs formes. Elles peuvent viser un ou plusieurs salariés, voire tous les salariés d'un atelier ou d'un établissement, ou encore le syndicat auquel appartient le militant ciblé ; elles sont donc de nature individuelle ou collective. Elles peuvent être formelles (organisation directe par l'employeur de canaux de consultations autres que syndicaux pour offrir un espace d'expression aux salariés) ou informelles, se déployant alors de manière plus ou moins ordonnée dans les pratiques quotidiennes de travail. Elles peuvent être préventives, réaffirmant ici la logique des stratégies anti-syndicales déployées au cours de la campagne de syndicalisation, ou répressives, visant à éliminer les syndicats ou à s'y substituer lorsqu'ils existent dans l'entreprise. Enfin, elles peuvent impliquer ou non des démarches d'ordre juridique (RIMBERT, CRESPO, 2004).

Les pratiques discriminatoires peuvent se manifester à différents moments de la carrière du militant visé (embauche, période d'essai, promotion, augmentation salariale, licenciement pour faute ou motif économique), ou au cours des différents cycles de la vie du syndicat (accréditation, première négociation, renouvellement de l'accord de convention collective, etc.). À l'instar de Rachel SPIRE (2006), nous distinguons également les mesures discriminatoires ponctuelles des mesures continues. Alors que les premières font référence à une forme de discrimination qui se manifeste à un moment particulier de la relation d'emploi, les secondes renvoient plutôt à l'idée que la discrimination va s'étendre sur une certaine période, souvent à partir de l'embauche ou de l'adhésion au syndicat. 


\section{Typologie des stratégies anti-syndicales}

Dans la lignée des travaux de Roy (1980), Dundon (2002), Gall (2004), MARTINELlo et YATES (2004) et BoIvin (2010), la typologie de LAROCHE, BERNIER et DuPUIS (2015) postule que les employeurs peuvent utiliser simultanément des pratiques suppressives ou substitutives. Elle permet également de considérer les stratégies patronales déployées non pas uniquement au moment de la reconnaissance du syndicat, mais également tout au long de la relation de négociation. Parce qu'elle permet à la fois de préciser les intentions visées par les employeurs au moment de déployer leurs stratégies et d'intégrer l'ensemble de celles qui ont été répertoriées dans les jurisprudences analysées, qu'elles comportent ou non un effet discriminatoire, cette typologie s'avère particulièrement utile dans le cadre de la présente recherche.

Ce cadre d'analyse propose de classer les stratégies anti-syndicales en quatre types de « campagnes » distinctes conçues et mises en œuvre par les employeurs, à savoir : 1) la campagne de cooptation, qui se caractérise par une amélioration (promise ou effective) des salaires et conditions de travail afin d'éviter la présence d'un syndicat ; 2) la campagne de contrôle, par laquelle l'employeur encourage la reconnaissance d'un syndicat modéré ou la création d'un syndicat maison avec lequel il négociera ; 3) la campagne de peur, qui instaure un climat d'hostilité par la menace de possibles représailles en cas de mouvement collectif ; et 4) la campagne de blocage, qui désigne le recours à des mesures administratives, juridiques ou dilatoires afin de faire obstacle à la reconnaissance du syndicat et à la conclusion d'une convention collective. Les campagnes de cooptation et de contrôle sont des stratégies dites de substitution, pour reprendre les expressions utilisées plus haut, alors que celles de peur et de blocage sont des stratégies de suppression.

La campagne de cooptation vise à éviter la syndicalisation de l'entreprise en montrant aux travailleurs qu'elle s'avère inutile pour améliorer leur sort. Avant que le syndicat n'obtienne son accréditation pour représenter les salariés de l'établissement concerné, l'employeur peut ainsi améliorer ou promettre d'améliorer les salaires et les conditions de travail dans l'espoir de diminuer la propension à la syndicalisation de ses travailleurs (DUNDON, GOLLAN, 2007 ; BRONFENBRENNER, 2009). Il peut aussi répondre à des doléances de longue date (GALL, 2004) ou améliorer l'environnement de travail (PIER, 2007). Cette stratégie peut encore être déployée une fois le syndicat accrédité. L'employeur tentera alors de le marginaliser en favorisant d'autres canaux, non syndicaux, de représentation, consultation et communication (DUNDON, 2002 ; DUNDON et al., 2006 ; PeETZ, 2002 ; GAlL, 2002, 2004 ; COOPER et al., 2009 ; GIRAUD, 2013). De telles pratiques constituent de bons exemples de formes inversées de discrimination syndicale. S'inspirant des travaux d'Étienne PenISSAT (2013), notre étude prend en considération ces formes de discrimination qui, sous prétexte de promouvoir la paix sociale au sein de l'organisation, proposent de nouvelles manières (non syndicales) d'organiser la parole collective des salariés. 
La campagne de contrôle regroupe les stratégies qui permettent à l'employeur de limiter les conséquences de l'accréditation d'un syndicat, pour lui ou son entreprise (GALL, 2004). Au Canada, le système de monopole de représentation syndicale et les contraintes imposées aux employeurs au cours du processus d'accréditation les limitent dans leurs velléités anti-syndicales. Cependant, certaines pratiques, comme le fait de favoriser l'accréditation d'un syndicat jugé « modéré », leur permettent tout de même d'exercer un certain contrôle sur le collectif salarié (GALL, 2002, 2004 ; DUNDON et al. 2006 et 2010 ; CoOPER et al., 2009 ; ClÉMENT, 2013 ; CHAPPE, 2015). Cette stratégie aboutit parfois à la conclusion d'un accord factice ne comportant que peu d'avantages pour les travailleurs (GALL, 2002 ; Dundon et al., 2006 et 2010). $\mathrm{Si}$ ces derniers décident de s'organiser, l'employeur préfère alors choisir le syndicat qu'il juge approprié et qui lui permet de limiter les conséquences de la syndicalisation. Dans un système comme celui de la France, cette tactique pourrait se traduire par la promotion d'un syndicalisme peu « combatif » ou même par des dispositifs favorisant une liste particulière au moment des élections professionnelles ou des négociations (Penissat, 2013). En déployant de telles stratégies, les employeurs prennent la voie de la discrimination syndicale inversée pour tenter de minimiser l'impact de la présence syndicale dans leur entreprise.

La campagne de peur, qui peut être mobilisée autant avant que pendant le processus d'accréditation, a pour objectif de saboter les tentatives de syndicalisation en instaurant un climat de peur et en menaçant de possibles représailles tout mouvement collectif. Le but de ces actions est triple : décourager les militants de demeurer actifs au sein du syndicat ; les empêcher d'organiser l'expression des travailleurs ; enfin, faire peur aux salariés afin d'affaiblir leur soutien aux représentants syndicaux (GALL, 2004). Des pratiques variées permettent de nourrir ce climat de craintes, les plus utilisées étant sans aucun doute l'intimidation, le harcèlement et le licenciement (ou les menaces d'y recourir) des militants syndicaux (BRONFENBRENNER, 1994, 1996 et 2009; COOPER et al., 2009 ; DEBRÉGEAS, 2013). Les employeurs ciblent les militants les plus actifs dans l'entreprise et les surveillent étroitement, ce qui les aide, dans certains cas, à trouver des motifs apparemment valables de licenciement (GALL, 2004 ; CLÉMENT, 2013). Parmi les autres moyens mis en œuvre, on relève également une surveillance accrue des salariés, la distribution de documents ou de vidéos anti-syndicaux, l'organisation de réunions obligatoires où sont évoqués les risques associés à la présence syndicale, la mise en place de rencontres individuelles avec les salariés, l'installation de comités anti-syndicaux, le dépôt de plaintes contre les syndicats pour pratiques déloyales, ainsi que la menace de fermeture ou la fermeture réelle des établissements (BRonfenbrenner, 2009 ; CoOPER et al., 2009 ; Pier, 2007 ; Dundon, 2002 ; Dundon et al., 2006 ; HuRD, UeHLEIN, 1994 ; RidDELL, 2001). En plus d'être les plus mobilisées par les employeurs, comme nous le verrons, ces pratiques sont les plus agressives et constituent de bons exemples de discrimination syndicale directe (représailles à l'encontre des militants en raison de leur engagement syndical, refus d'embaucher ou de considérer la candidature d'un travailleur en raison de son affiliation syndicale, etc.). 
La campagne de blocage fait référence à diverses stratégies, qui sont également mises en œuvre avant ou après l'accréditation d'un syndicat dans l'entreprise. Elles cherchent précisément à empêcher sa reconnaissance effective par des obstacles freinant ou bloquant la conclusion d'un accord collectif (BoIvin, 2010 ; GALL, 2002). Les employeurs ont ainsi très souvent recours à des consultants ou à des avocats spécialisés dans les techniques d'évitement syndical, afin de contrecarrer les campagnes lors desquelles les travailleurs tentent de s'organiser collectivement (GALL, 2002 ; JULLIARD, 2013), ou à différents moyens administratifs pour entraver le processus d'accréditation (THOMASON, 1994 ; GALL, 2002). Ils peuvent aussi, directement, tenter d'inciter les travailleurs à démissionner du syndicat avant le dépôt de la requête en accréditation, faire signer des pétitions par certains salariés pour montrer leur opposition à la syndicalisation ou même utiliser la sous-traitance pour diviser le collectif des salariés (GALL, 2004). Dans ce cadre, certaines directions d'entreprise ou d'établissement limitent l'accès des représentants du syndicat aux sites de travail, ou empêchent les militants d'y solliciter les travailleurs (PEETZ, 2002).

D'autres pratiques concrètes - comme refuser de communiquer avec les représentants syndicaux et de participer aux réunions (GALL, 2004), mettre en place des négociations soit purement formelles, soit adoptant des techniques de négociation dure ou reposant sur de la mauvaise foi (HURD, UEHLEIN, 1994) - servent à empêcher la conclusion d'un accord collectif. Les employeurs peuvent encore tenter de diviser les salariés, par exemple en offrant des promotions et des privilèges à certains représentants ou en propageant des rumeurs sur d'autres. Ces pratiques, souvent de nature collective, sont également discriminatoires : il n'y a qu'à penser aux nombreuses représailles (mises à pied, licenciements, etc.) réservées aux sympathisants syndicaux, ou encore à l'embauche ciblée de travailleurs anti-syndicaux, en vue d'affaiblir la représentativité syndicale.

\section{Discrimination syndicale : quelle protection législative ?}

Dans le cadre de notre étude, nous avons retenu deux provinces : le Québec et l'Ontario. Le choix de ces juridictions s'explique par l'importance accordée à la négociation collective comme mécanisme de régulation dans leur système de relations industrielles, où l'acteur syndical est réputé jouer un rôle dynamique (THOMPSON $e t$ al., 2003). Le cas de l'Ontario se justifie également par la possibilité dont dispose la commission des relations du travail ${ }^{3}$ de compenser le recours patronal à des pratiques déloyales par l'accréditation directe du syndicat (WILlows, SCHETAGNe, 2011). Le cas du Québec a quant à lui été ciblé parce que la portée des articles de loi visant à prohiber

\footnotetext{
3. Les commissions des relations du travail sont des tribunaux administratifs dont le mandat est de veiller à l'application des lois encadrant les rapports collectifs du travail. Elles tranchent également les litiges qui en découlent. Chaque province canadienne dispose d'un tribunal de ce type.
} 
la mise en œuvre de telles stratégies y a été élargie. En plus de permettre les recours devant les juridictions pénales, les dispositions légales offrent désormais la possibilité d'obtenir des compensations en droit civil, ce qui peut les rendre plus intéressants aux yeux des salariés. Ajoutons que peu de chercheurs canadiens se sont intéressés aux décisions québécoises en la matière, en raison notamment de la barrière de la langue.

$\mathrm{Si}$, tant au Québec qu'en Ontario, plusieurs textes juridiques régissent les relations collectives de travail, les deux provinces possèdent un régime général de rapports collectifs qui présente plusieurs similitudes. Au Québec, le régime général est prévu par le Code du travail adopté en 1964. Son pendant ontarien est la Loi de 1995 sur les relations de travail (Labour Relations Act). Ces deux régimes sont fortement inspirés du Wagner Act américain (RouILlard, 2004 ; TRUdEAU, VeILleuX, 1995) et ont pour objectifs « de reconnaître et de protéger le droit d'association des salariés, le droit à la négociation collective, de favoriser l'amélioration des conditions de travail tout en prenant en compte les intérêts économiques de l'entreprise ainsi que la santé et la sécurité du public et de préserver la paix industrielle » (TANGUAY-LAVALLÉE et al., 2012, p. 4 ; art. 2 Loi de 1995 sur les relations de travail). Le tableau ci-après résume certaines caractéristiques du régime général de rapports collectifs de ces deux provinces.

\section{TABLEAU - Caractéristiques des régimes juridiques encadrant les rapports collectifs de travail} au Québec et en Ontario

\begin{tabular}{|c|c|c|}
\hline Caractéristiques & Québec & Ontario \\
\hline Processus d'accréditation & $\begin{array}{l}\text { - Automatique lorsque plus de } \\
50 \% \text { des membres de l'unité } \\
\text { d'accréditation* ont adhéré au } \\
\text { syndicat. } \\
\text { - Vote obligatoire si de } 35 \text { à } 50 \% \\
\text { des membres de l'unité ont adhéré } \\
\text { au syndicat. Si plus de } 50 \% \text { des } \\
\text { membres votent alors pour le } \\
\text { syndicat, il est accrédité. }\end{array}$ & $\begin{array}{l}\text { - Si } 40 \% \text { ou plus des membres de } \\
\text { l'unité d'accréditation ont adhéré au } \\
\text { syndicat, un scrutin est tenu. } \\
\text { - Si plus de } 50 \% \text { des membres } \\
\text { appuient le syndicat, il est accrédité. }\end{array}$ \\
\hline Cotisations syndicales & \multicolumn{2}{|c|}{ - L'employeur doit prélever les cotisations syndicales à la source. } \\
\hline Négociation collective & \multicolumn{2}{|c|}{$\begin{array}{l}\text { - Obligation de négocier de bonne foi. } \\
\text { - Les deux parties (patronale et syndicale) peuvent demander la nomination } \\
\text { d'un arbitre si elles n'arrivent pas à conclure un premier accord collectif. }\end{array}$} \\
\hline Droit de grève & \multicolumn{2}{|c|}{$\begin{array}{l}\text { - Interdiction de recourir à la grève pendant toute la durée de la convention } \\
\text { collective. }\end{array}$} \\
\hline
\end{tabular}

* L'unité d'accréditation est le collectif de salariés (qui peut correspondre à une équipe de travail, un service, un atelier, une usine, l'ensemble d'un site ou d'un établissement) qui demande à être représenté par un syndicat en vue de conclure un accord collectif précisant de nouvelles modalités de rémunération et, plus largement, les conditions d'emploi, de travail et de protection sociale.

Source : synthèse originale par les auteures du Code du travail du Québec (1964) et du Labour Relations Act de 1'Ontario (1995). 
Concernant plus spécifiquement les tactiques anti-syndicales, y compris celles qui ont un effet discriminatoire, le Code du travail du Québec et la Loi de 1995 sur les relations de travail en Ontario prévoient un éventail de mesures, dont les dispositions les plus intéressantes sont les articles 12 à 14 et 15 à 17 du Code du travail au Québec et les articles 70, 72 et 76 de la Loi de 1995 sur les relations de travail. Ces dispositions législatives prohibent l'ingérence et la mainmise de l'employeur sur une association de salariés ainsi que les discriminations et représailles en raison d'activités syndicales ou de l'exercice d'un autre droit protégé. Elles encadrent par ailleurs la liberté d'expression de l'employeur, en interdisant notamment l'intimidation de la part des syndicats. L'encadré qui suit présente plus en détail chacune de ces dispositions.

\section{ENCADRÉ 1}

\section{Résumé des dispositions législatives étudiées}

\section{Entrave et contrôle des syndicats}

Au Québec, il est interdit à l'employeur de prendre le contrôle d'une association de salariés (art. 12 du Code du travail). Sont considérés comme des indices d'une prise de contrôle le fait que l'employeur finance une association, qu'il en favorise une plutôt qu'une autre ou qu'il s'ingère dans le choix des dirigeants syndicaux ${ }^{1}$. En Ontario, l'employeur ne peut pas non plus participer à la formation, à l'administration ou au financement d'un syndicat. Il lui est aussi interdit de choisir un syndicat au détriment d'un autre (art. 70 de la Loi de 1995 sur les relations de travail).

\section{Liberté d'expression et intimidation}

Au Québec, l'employeur ne peut mentir, menacer ni faire de promesses afin de décourager l'adhésion syndicale (art. 12 du Code du travail). Les salariés doivent être libres de l'écouter ou non. Les réunions obligatoires (payées ou non) ou pendant les heures de travail sont à éviter ${ }^{2}$. Il est interdit de recourir à l'intimidation pour empêcher la syndicalisation (art. 13 du Code du travail). Il y a intimidation si les paroles et actions de l'employeur créent une appréhension réelle chez le travailleur, que cette crainte l'ait dissuadé ou non de devenir membre de l' association de salariés ${ }^{3}$. En Ontario, l'employeur est libre d'exprimer son point de vue, pourvu qu'il n'use pas de la contrainte, de l'intimidation, de la menace, d'une promesse, ni n'abuse de son influence (art. 70 de la Loi de 1995 sur les relations de travail). Il y a également interdiction de recourir à l'intimidation pour empêcher la syndicalisation ou l'exercice d'un autre droit prévu par la loi (art. 76 de la Loi de 1995 sur les relations de travail).

1. Syndicat des employés de Mauricie Toyota c. Syndicat national de l'automobile, de l'aérospatiale, du transport et des autres travailleurs et travailleuses du Canada (TCA-Canada), 2007 QCCRT 0317 [Toyota].

2. Syndicat canadien des communications, de l'énergie et du papier, section locale 194 c. Disque Americ Inc. [1996] T.T. 451.

3. Lagacé c. Laporte, [1983] T.T. 354, [1983] AZ-83147103 (T.T.). 


\section{Représailles et discrimination}

Au Québec, il est interdit de discriminer ou de sanctionner un salarié exerçant un droit protégé par la loi. Il existe deux types de protection :

- l'article 14 du Code du travail prévoit que l'employeur ne peut refuser d'embaucher un travailleur ou le menacer de sanctions disciplinaires à cause de ses activités syndicales ${ }^{4}$. Ce recours est surtout utilisé dans le cas de refus d'embauche (Coutu et al., 2013);

- l'article 15 du Code prévoit qu'un employeur qui « congédie [licencie pour faute], suspend ou déplace un salarié, exerce à son endroit des mesures discriminatoires ou de représailles, ou lui impose toute autre sanction à cause de l'exercice par ce salarié d'un droit qui lui résulte du présent Code » commet une pratique interdite.

Si ces recours présentent des similitudes, il existe une différence fondamentale entre les deux. Contrairement à l'article 14 où le syndicat doit prouver l'intention anti-syndicale de l'employeur, le recours initié sous l'article 15 du Code établit une présomption favorable au salarié. Ainsi, dès qu'il est démontré que le salarié sanctionné exerçait un droit protégé par le Code, il y a un renversement de la charge de la preuve et c'est l'employeur qui doit démontrer la légalité de la sanction contestée (art. 17 du Code) ${ }^{5}$.

En Ontario, il n'est pas non plus permis de refuser d'employer une personne ou de la discriminer dans l'emploi. L'employeur ne peut menacer de sanctions ou sanctionner un salarié qui exerce son droit d'association. De plus, l'inclusion d'une clause limitant le droit d'association dans le contrat de travail est expressément interdite (art. 72 de la Loi de 1995 sur les relations de travail). Finalement, lorsqu'un salarié dépose une plainte, c'est à l'employeur qu'il revient de démontrer qu'il a une cause juste et suffisante pour licencier ou sanctionner le plaignant (art. 96 [5] de la Loi).

4. Respectivement : Boivin c. Comité conjoint de l'industrie et de la confection pour dames au Québec (1981) T.T. et Fleurent c. Compagnie Wal-Mart du Canada, 2005 QCCRT 0095.

5. Cette situation est similaire à celle désormais observée en France, comme le rappelle le dernier numéro de la revue Travail et Emploi (AMOssé, DenIs, 2016).

\section{Considérations méthodologiques et corpus d'analyse}

Les tactiques anti-syndicales ont été peu étudiées au Québec. C'est particulièrement vrai en ce qui concerne la discrimination à l'égard des sympathisants et militants syndicaux. Pour les documenter, nous avons procédé à une analyse jurisprudentielle des décisions rendues sur la base des articles 12 à 15 du Code du travail du Québec. Suivant une perspective comparative, nous avons également examiné les décisions portant sur les articles 70, 72 et 76 de la Loi de 1995 sur les relations de travail de l'Ontario. Pour les fins de notre analyse, n'ont été retenus que les cas où le recours à une tactique anti-syndicale par l'employeur - soit directement, soit par l'intermédiaire de salariés ou d'un syndicat rival - a été sanctionné par le tribunal spécialisé compétent. Ces décisions ont été sélectionnées grâce à deux bases de données, Soquij (Québec) et CanLII (Ontario), qui publient les décisions des commissions des relations du travail. Notre méthodologie est présentée dans l'encadré 2. 


\section{ENCADRÉ 2}

\section{Méthodologie de la recherche}

Notre analyse porte sur les décisions où le recours à une tactique anti-syndicale par l'employeur a été sanctionné par le tribunal spécialisé compétent. Ne tenant compte que des cas où l'infraction a été judiciarisée et effectivement reconnue, ce travail comporte certaines limites, dont celle de ne pas permettre, dans un premier temps, d'étudier le rôle des tribunaux spécialisés dans l'application et l'effectivité du droit des rapports collectifs. Elle permet cependant de documenter le recours à des tactiques anti-syndicales au Canada, et particulièrement au Québec où ce sujet a jusqu'à présent été peu abordé.

L'analyse de ces décisions se référera aux tactiques identifiées lors de notre revue de littérature. Toutes ne seront toutefois pas observées : en effet, certaines tactiques identifiées dans les travaux de relations industrielles ne sont pas sanctionnées par la loi : par exemple, promettre d'améliorer les salaires, utiliser des consultants, etc., n'a rien d'illégal.

Les décisions étudiées contiennent habituellement les éléments suivants : énumération des faits pertinents relatifs à la plainte, prétentions des parties et analyse de la plainte par la commission. La plupart du temps, les décisions font peu état du contexte syndical général dans l'entreprise et de l'historique des plaintes déjà déposées contre l'employeur. Leur longueur varie de quelques pages à une centaine de pages, la plupart faisant entre dix et quinze pages. Concernant les motifs de la décision, certains commissaires analysent globalement l'ensemble des faits soumis pour conclure à une pratique interdite par la loi, alors que d'autres identifient clairement les actes reprochés.

La recherche couvre une période de dix ans, de 2002 à 2012, ce qui permet notamment d'apprécier l'effet d'évolutions importantes dans le contexte institutionnel de chaque province ciblée : pensons par exemple aux changements d'orientation politique des gouvernements ou encore à la modification des pouvoirs de la commission des relations du travail du Québec en 2002 (Coutu et al., 2013).

Au total, nous avons recensé 145 cas d'entreprises, 108 au Québec et 37 en Ontario, dans lesquels les tribunaux compétents ont reconnu que les employeurs avaient eu recours à une ou plusieurs stratégies anti-syndicales. Cette plus forte représentation des décisions en provenance du Québec dans le corpus d'analyse peut s'expliquer par exemple par la différence de précision des bases de données. Pour chacun des cas retenus, nous avons procédé à une analyse de contenu à partir d'une grille de lecture identifiant les types de stratégies anti-syndicales recensées dans la littérature. Par souci méthodologique, chacun des 145 cas a été analysé par deux personnes, conférant ainsi plus de rigueur au processus. 


\section{Analyse des résultats}

Cette partie présente les principaux constats qui émergent de notre analyse jurisprudentielle. Mentionnons d'entrée de jeu que dans un même cas l'employeur a pu utiliser plusieurs campagnes et tactiques anti-syndicales ${ }^{4}$ et à différents moments, ce qui constitue un indice de son degré d'opposition à la syndicalisation. Notre recherche a permis de répertorier 385 tactiques anti-syndicales dans les 145 cas d'entreprises du corpus d'analyse. L'analyse qui suit se concentre toutefois sur un nombre restreint de cas, plus précisément 125 cas d'entreprises (24 en Ontario et 101 au Québec), dans lesquels des tactiques impliquant une forme de discrimination syndicale à l'égard des militants ont été identifiées : au total, 95 tactiques discriminatoires y ont été identifiées en Ontario, et 236 au Québec.

\section{Campagnes et tactiques utilisées}

Peu d'employeurs ont eu recours aux tactiques correspondant aux campagnes de cooptation dans notre échantillon ( 7 cas, 11 tactiques), lesquelles ne sont de fait que rarement considérées comme des stratégies discriminatoires envers les militants concernés (et donc, peu présentes à ce titre dans le corpus). Lorsqu'ils lancent ce type de campagne, les employeurs promettent le plus souvent d'améliorer les conditions de travail et les salaires ( 7 cas sur 7). De telles promesses ont été utilisées tant pour inciter les salariés à ne pas adhérer à un syndicat que pour les pousser à se désyndiquer ${ }^{5}$. Cette tactique est porteuse d'une forme inversée de discrimination, les employeurs offrant alors un traitement différencié aux salariés qui cherchent à se syndiquer ou à le demeurer, non pas pour punir l'adhésion, mais pour récompenser la non-adhésion. Par exemple, un employeur a décidé, au lendemain du dépôt d'une requête en accréditation, d'augmenter les salaires de tous les salariés de l'entreprise, sauf de l'organisateur de la campagne syndicale qui s'est également vu confier des tâches moins importantes qu'auparavant ${ }^{6}$. Dans un autre cas, après le dépôt de la requête en accréditation, l'employeur a octroyé de substantielles augmentations du taux de salaire horaire, allant jusqu'à 10 dollars pour certains travailleurs. Il a également permis à certains d'entre eux d'utiliser les véhicules de la compagnie, ce qu'il n'avait jamais fait auparavant. La commission des relations de travail a alors reconnu que l'employeur cherchait à

\footnotetext{
4. Les tactiques sont des moyens concrets mis en œuvre par les employeurs pour lutter contre les syndicats. Elles peuvent s'inscrire dans un seul ou plusieurs des quatre types de campagnes anti-syndicales que nous avons présentées auparavant (cooptation, contrôle, peur, blocage).

5. Voir par exemple : Travailleuses et travailleurs unis de l'alimentation et du commerce, section locale 501 c. Charcuterie Tour Eiffel Inc. (division Charcuterie de Bretagne), 2003 QCCRT 0676, DTE 2004T-190 — regroupée avec Travailleurs et travailleuses unis de l'alimentation et du commerce, section locale 501 c. Charcuterie Tour Eiffel Inc. (division Charcuterie de Bretagne), 2004 QCCRT 0028 [cas Tour Eiffel].

6. Ontario Pipe Trades Council of the United Association of Journeymen and Apprentices of the Plumbing and Pipefitting Industry of the United States and Canada v. Eagle Plumbing Contractors Inc., 2012 CanLII 47363 (CRT Ont.) [cas Eagle Plumbing Contractors Inc.].
} 
influencer les travailleurs en prévision du vote d'accréditation qui allait avoir lieu ${ }^{7}$. Dans un autre cas, l'employeur a pris la décision d'améliorer les conditions de travail dans ses établissements non syndiqués dans l'unique but de montrer l'inutilité du syndicat à ses salariés syndiqués d'un autre établissement couvert par un certificat d'accréditation ${ }^{8}$. Ainsi, les stratégies anti-syndicales et discriminatoires peuvent avoir un effet au-delà des unités d'accréditation qu'elles visent directement.

Une autre forme de discrimination syndicale inversée a été identifiée dans deux des cas de notre corpus : les employeurs y mettent en place, en marge des activités syndicales, des mécanismes d'expression et de consultation pour les salariés ${ }^{9}$. Ces mécanismes alternatifs de représentation peuvent être efficaces pour convaincre les travailleurs de s'éloigner des canaux syndicaux traditionnels. Ils portent sur les conditions de travail et revendications immédiates des travailleurs, et sont présentés comme des formes positives de résolution des problèmes, contrairement à la vision conflictuelle généralement associée aux syndicats.

Que faut-il conclure du nombre relativement restreint de cas où la campagne de cooptation a été utilisée ? Est-ce le signe que les employeurs la jugent inefficace et préfèrent s'en remettre à des campagnes plus agressives ? Ce constat nous amène à discuter d'une des limites de notre étude. Notre corpus d'analyse se concentre en effet sur les cas où non seulement les pratiques déloyales de l'employeur ont été judiciarisées, mais aussi sur ceux où elles ont été sanctionnées. Les tactiques de cooptation étant moins agressives, de nombreux cas où cette stratégie a été mobilisée par l'employeur ont sans doute échappé à notre analyse.

Les campagnes de contrôle, lesquelles consistent pour l'employeur à empêcher la formation d'une association collective de salariés, ou à avoir la mainmise sur elle, sont aussi présentes dans un nombre limité de cas de notre corpus (22, pour 23 tactiques). Notons que notre analyse nous a amenées à élargir la définition de ce type de campagne afin de compléter la liste des tactiques documentées dans les travaux antérieurs. De telles tactiques permettent à l'employeur de contenir la « contestation » dans son organisation. Par exemple, l'employeur peut chercher à entraver l'expression de revendications des salariés par un contrôle inhabituel des décharges syndicales ${ }^{10}$

7. International Brotherhood of Electrical Workers, Local 353 v. Aerostar Electrical Services Inc., 2009 CanLII 9205 (CRT Ont.).

8. Syndicat des quincailleries et commerces de Québec Inc. (CSD) c. Canac-Marquis Grenier Ltée et Paul-A. Audet, 2005 QCCRT 0297 ; Soquij AZ-50315469. Suivi de la décision : Canac-Marquis Grenier Ltée c. Syndicat des quincailleries et commerces de Québec Inc. (CSD), 2005 QCCRT 0394 ; Soquij AZ-50323580 (Requête en sursis d'exécution rejetée) ; Canac-Marquis Grenier Ltée c. Commission des relations du travail, Soquij AZ-50325675 (Requête en sursis d'exécution accueillie) ; Canac-Marquis Grenier Ltée c. Commission des relations du travail, Soquij AZ-50778076 (Requête en révision judiciaire accueillie en partie) ; Canac-Marquis Grenier Ltée et Syndicat des quincailleries et commerces de Québec Inc. (CSD), 2005 QCCRT 0663 ; Soquij AZ-50346532 (Requête en révision accueillie en partie) [Cas Canac-Marquis].

9. Voir par exemple : Bernard c. Métallurgistes unis d'Amérique, local 9414 Syndicat et Les microcircuits C-Mac (Sélectron), 2003 QCCRT 0406.

10. Elles sont appelées « libérations syndicales » au Québec. Les salariés ou le syndicat qui veulent se plaindre de la manière dont l'employeur applique ou interprète la convention collective peuvent soumettre une plainte officielle, 
$\left(4 \operatorname{cas}^{11}\right)$, inciter les salariés à retirer leurs griefs ${ }^{12}$, voire demander la révocation d'une accréditation syndicale afin de nuire au traitement de revendications toujours pendantes à la suite de la fermeture de l'entreprise ${ }^{13}$. L'employeur peut tenter plus largement de limiter la liberté d'expression du syndicat ${ }^{14}$, par exemple en l'empêchant d'informer les salariés sur les projets qu'il mène, ce qui lui évite de voir sa réputation ternie. Dans 7 cas, l'employeur a essayé de favoriser l'accréditation d'une organisation plutôt que d'une autre, ou s'est assuré qu'un syndicat modéré soit accrédité. Ce faisant, ces employeurs ont privilégié la voie de la discrimination, sous une forme inversée, pour favoriser un syndicat moins combatif et plus conciliant à l'égard des intérêts patronaux. Une telle préférence peut se manifester de différentes manières : laisser circuler les représentants syndicaux d'une organisation donnée dans l'entreprise tout en interdisant la présence des représentants d'un autre syndicat ${ }^{15}$; fermer les yeux sur les intimidations exercées par le syndicat que les employeurs entendent avantager ; régler rapidement les revendications qu'il porte afin de le valoriser par rapport à un syndicat plus combatif ${ }^{16}$; surveiller et exercer des représailles contre des travailleurs contestataires, qui désirent un changement de syndicat au sein de l'entreprise, etc. Dans certains de nos cas, l'employeur a poussé plus loin la discrimination en cherchant à faire accréditer un « syndicat maison » $\left(4 \operatorname{cas}^{17}\right)$. Un exemple montre qu' un employeur a même tenté d'inclure des cadres et des membres de sa famille immédiate dans l'unité de négociation de manière à contrôler ou à minimiser la contestation ${ }^{18}$.

Les campagnes de peur sont de loin les plus utilisées par les employeurs dans notre corpus d'analyse ( 105 cas, pour un total de 200 tactiques), que ce soit avant ou après l'accréditation syndicale. Ces tactiques ne s'adressent pas uniquement aux militants syndicaux : les sympathisants syndicaux, les travailleurs exerçant leurs droits (par exemple, celui de déposer un grief), ainsi que tous les travailleurs qui pourraient être tentés de se syndiquer sont aussi visés. Les campagnes de peur regroupent des tactiques comme la distribution de documents anti-syndicaux aux salariés ( 19 cas, dont deux où

c'est-à-dire un grief, à l'arbitrage. Bien que le grief soit souvent déposé par un salarié, il appartient au syndicat de décider d'y donner suite ou non. Les représentants du syndicat doivent donc pouvoir disposer de temps (décharges, ou libérations, syndicales) afin de pouvoir adéquatement représenter les salariés et défendre leur grief.

11. Voir par exemple : Syndicat des professionnel.le.s en soins infirmiers et cardio-respiratoires de Drummondville (FIQ) c. Centre de santé et de services sociaux Drummond, 2010 QCCRT 0613.

12. Ibid.

13. 3539491 Canada Inc. c. Syndicat des Métallos, section locale 2843, 2012 QCCRT 0028.

14. Voir par exemple : Syndicat des employée.es de techniques professionnelles et de bureau d'Hydro-Québec, section locale 2000, Syndicat canadien de la fonction publique c. Hydro-Québec, 2012 QCCRT 0084 et Desfossés c. Société de transport de Sherbrooke et Commission des relations du travail, 2011 QCCA 119 (rétablissant 2007 QCCRT 0556, DTE 2007T-1012 ; infirmant 2009 QCCS 600, DTE 2009T-195 [rectifié le 24 février 2009]).

15. Cas Canac, supra note 8.

16. Syndicat canadien des communications, de l'énergie et du papier (SCEP), section locale 197 c. Solive Ajourée 2000 Inc. et Syndicat des employés de S.A. 2000, 2007 QCCRT 0582.

17. Voir par exemple : Syndicat des employés de Camtruck c. Camtruck, 2011 QCCRT 456.

18. Labourers' International Union of North America, Ontario Provincial District Council v. Swift Railroad Contractors Corporation, 2011 CanLII 36837 (CRT Ont.) [cas Swift Railroad]. 
l'employeur fait livrer les missives par huissier ${ }^{19}$ ), les réunions obligatoires d'information anti-syndicale $\left(16 \mathrm{cas}^{20}\right)$, les rencontres permettant à l'employeur d'isoler de petits groupes de salariés pour leur faire part de sa position sur la syndicalisation $\left(5 \mathrm{cas}^{21}\right)$ ou encore, bien que plus rare, la mise sur pied de comités anti-syndicaux $\left(3 \operatorname{cas}^{22}\right)$.

Parmi les tactiques répertoriées, 182 peuvent être considérées comme discriminatoires (91\%). Les employeurs ont, dans certains cas, surveillé les militants (11 cas), sont parfois allés jusqu'à les interroger (12 cas) ou constituer des « listes noires » (blacklists) de travailleurs syndiqués (5 cas). D'autres employeurs agissent de telle sorte que les travailleurs impliqués dans une activité syndicale sont amenés à craindre de perdre certains avantages : dans 8 cas, les employeurs ont en effet utilisé divers avertissements relatifs à la sécurité de l'emploi en cas de syndicalisation, au choix d'un syndicat en particulier ou même au maintien de l'accréditation. D'autres employeurs encore vont plus loin, menaçant directement de fermer leur entreprise en cas de syndicalisation (10 cas).

Dans 11 des cas analysés, les militants syndicaux ont reçu des intimidations plus graves de la part de l'employeur ou de ses représentants. Dans un cas par exemple, les militants ont été directement menacés de violence physique, les travailleurs non syndiqués étant encouragés à frapper les responsables syndicaux par l'octroi d'une récompense ${ }^{23}$. Dans un autre cas, ce sont des menaces de mort qui ont été adressées aux responsables syndicaux extérieurs à l'entreprise ${ }^{24}$ ! Ces menaces deviennent de plus parfois bien réelles et se transforment en gestes violents afin d'intimider les travailleurs. Sur un chantier de construction par exemple, un contremaître ayant aperçu deux responsables syndicaux leur a ordonné de quitter les lieux et, constatant qu'ils ne s'exécutaient pas, a utilisé une grue pour suspendre leur véhicule dans les airs. Il n'a relâché le véhicule, non sans dommages, qu'une fois qu'il a appris que la police était en route ${ }^{25}$. Dans un autre cas, un représentant de l'employeur, à la suite du licenciement

19. Syndicat national des travailleuses et travailleurs des pâtes et cartons de Jonquière Inc. (usine pâte) (CSN) et Cascades Fjordcell, division de Cascades Canada Inc., 2005 QCCRT 0132 (annulée par 2006 QCCS 1815 ; permission d'appeler accueillie en partie : 2006 QCCA 754 [2006] ; appel accueilli : 2007 QCCA 1765).

20. Bernard c. Métallurgistes unis d'Amérique, local 9414 Syndicat et Les microcircuits C-Mac (Sélectron), 2003 QCCRT 0406.

21. Voir par exemple le cas Tour Eiffel, supra note 5.

22. Voir par exemple : Syndicat des travailleuses et travailleurs du Mount Stephen Club-CSN Requérant c. Mount Stephen Club (9166-1389 Québec Inc.) et 9166-9093 Québec Inc., 2011 QCCRT 0499 — regroupée avec Syndicat des travailleuses et travailleurs du Mount Stephen Club-CSN c. Mount Stephen Club (9166-1389 Québec Inc.) et 9166-9093 Québec Inc. et Alain Major, 2012 QCCRT 0005.

23. Labourers' International Union of North America, Local 1059 v. East Elgin Concrete Forming Limited, 2007 CanLII 29221 (CRT Ont.).

24. Labourers' Union of North America, Ontario Provincial District Council v. Complete Excavating Limited, 2010 CanLII 19768 (CRT Ont.).

25. Ibid. 
du représentant syndical de l'entreprise, a arraché des mains d'un travailleur sa carte de membre du syndicat et l'a brûlée devant lui ${ }^{26}$.

Dans 88 des cas, des tactiques telles que l'intimidation, le harcèlement, les représailles et le renvoi des militants syndicaux ont été mobilisées par l'employeur. Ces représailles prennent de multiples formes et sont d'intensité variable. Le simple fait que deux des trois membres du comité exécutif d'un syndicat aient affiché publiquement leur appui à un syndicat rival, sans doute jugé plus revendicatif par l'employeur, leur a valu une suspension ${ }^{27}$. Dans certains cas, les tactiques discriminatoires s'attaquent à des cibles jugées plus faciles, comme les travailleurs précaires. Un employeur a ainsi pris la décision de licencier une employée en période d'essai au cours d'une négociation parce qu'elle avait participé à une action organisée par le syndicat, au cours de laquelle elle portait un pull à l'effigie du syndicat ${ }^{28}$. Dans certaines circonstances, l'opposition de certains employeurs à la syndicalisation est si forte qu'ils n'hésitent pas à licencier les militants syndicaux. Ces pratiques discriminatoires peuvent être mises en œuvre très rapidement après que l'employeur a été informé de l'implication d'un travailleur dans un processus de syndicalisation ${ }^{29}$.

Les mesures de représailles peuvent également viser plus d'un salarié de l'entreprise. Elles consistent parfois en des menaces envers l'ensemble des salariés qui ont une carte syndicale ${ }^{30}$, mais aussi en des mises à pied massives de travailleurs d'unités syndiquées, comme celle imposée à 162 ouvriers le jour où ils ont été convoqués à une assemblée syndicale pour la tenue d'un vote de grève ${ }^{31}$. Dans un autre cas, un employeur a décidé en pleine campagne de syndicalisation d'externaliser une importante partie de ses activités de production et d'emballage en Chine, et a procédé au licenciement d'une grande partie de ses effectifs, dont cinq des six responsables syndicaux et environ $85 \%$ des adhérents au syndicat ${ }^{32}$. Un autre employeur a préféré encourager la démission de plusieurs de ses travailleurs en modifiant substantiellement leurs conditions de travail ${ }^{33}$.

Compte tenu de la gamme étendue des tactiques qui sont associées à la campagne de peur, de la variété des moments où elles peuvent être mobilisées et des nombreuses personnes qu'elles visent, rien d'étonnant à ce que leur efficacité soit reconnue dans

\footnotetext{
26. United Brotherhood of Carpenters and Joiners of America, Local 2486 v. Southend Drywall \& Acoustics Ltd., 2010 CanLII 13074 (CRT Ont.).

27. Teamsters/Conférence des communications graphiques, section locale 555 M c. Joncas Postexperts Inc. et Syndicat des travailleurs en expédition postale, 2008 QCCRT 0249.

28. Parenteau c. Villa du Boisé Inc., 2010 QCCRT 0591.

29. Universal Workers' Union, Labourers' International Union of North America Local 183 v. Battano Construction Ltd., 2005 CanLII 18259 (CRT Ont.).

30. Labourers' International Union of North America, Local 1059 v. Carlos Barbosa Concrete Limited, 2008 CanLII 26617 (CRT Ont).

31. Syndicat des employés manuels de la Ville de Québec, section locale 1638 - Syndicat canadien de la fonction publique c. Ville de Québec, 2012 QCCRT 0198.

32. United Steelworkers of America v. Extrufix-a Division of CPI Plastic Group Ltd., 2005 CanLII 25339 (CRT Ont.).

33. Voir le cas Swift Railroad, supra note 18.
} 
plusieurs travaux. Ces tactiques permettent de révéler les multiples facettes que prennent les pratiques de discrimination syndicale.

Enfin, les campagnes de blocage sont aussi fortement utilisées par les employeurs de notre échantillon, 85 d'entre eux ayant eu recours à 145 tactiques relevant de ce type de campagnes. La tactique la plus répandue est celle de la négociation de mauvaise foi (21 cas), qui se manifeste par exemple lorsque l'employeur nie le rôle de négociateurs qu'ont en principe les représentants syndicaux et qu'il transmet ses offres directement aux salariés, soit pour les inciter à faire pression sur leurs représentants syndicaux, soit pour miner leur confiance en eux ${ }^{34}$. Notons que le refus d'obtempérer aux demandes des représentants syndicaux, par exemple en refusant de leur fournir la liste des salariés, a également été observé dans $16 \mathrm{cas}^{35}$. La mobilisation de différents moyens dilatoires et recours légaux, allant par exemple du report de rencontres ou de réunions à la contestation de la compétence des instances administratives ou de la représentativité syndicale, revient également à plusieurs reprises (18 cas).

D'autres pratiques sont aussi utilisées par l'employeur et constituent des formes variées de discrimination syndicale, souvent de nature collective, notamment lorsque l'employeur tente d'affecter la représentativité syndicale, que ce soit par des mises à pied, par l'embauche de travailleurs qui sont contre la syndicalisation ou par le licenciement d'une partie des salariés couverts par le syndicat (6 cas). Cette dernière possibilité est parfaitement illustrée par un cas où l'employeur a supprimé 76 postes occupés par des salariés syndiqués, ce qui a privé le syndicat d'une partie importante de ses représentants dans un contexte de renouvellement de la convention collective ${ }^{36}$. Notons qu'ici, les suppressions ne se sont pas faites dans un contexte de manque de travail, puisque l'employeur a parallèlement créé un nombre équivalent de postes non syndiqués. Le recours à la sous-traitance ou à des agences de placement peut aussi constituer une pratique discriminatoire, lorsqu'elle est utilisée pour diviser la main-d'œuvre ou écarter les équipes couvertes par un syndicat (4 cas). Dans un cas, l'employeur a demandé à une agence de placement de réaffecter, chez un autre employeur, deux travailleurs ayant une carte syndicale de manière à les isoler et à faire diminuer le nombre de salariés couverts par l'accord collectif ${ }^{37}$. Un autre employeur a offert du travail à des salariés suspendus uniquement s'ils acceptaient de réintégrer leur emploi à titre de sous-traitants non syndiqués ${ }^{38}$.

D'autre cas montrent encore que les employeurs incitent les travailleurs à démissionner du syndicat de différentes façons (10 cas) : par exemple, après avoir licencié

\footnotetext{
34. Voir par exemple : Syndicat canadien de la fonction publique, section locale 4290 c. Municipalité de Sainte-Béatrix, 2004 QCCRT 0527.

35. Voir par exemple : Travailleurs unis du pétrole du Canada, section locale 121 du Syndicat canadien des communications, de l'énergie et du papier c. Shell Canada Ltée, 2010QCCRT 0558.

36. Association des pompiers professionnels de Québec Inc. c. Ville de Québec, 2010 QCCRT 0210.

37. United Food and Commercial Workers' International Union (UFCW Canada) v. PPG Canada, Liberty Staffing Services Inc. and The Staffing Edge Inc., 2009 CanLII 15058 (CRT Ont.).

38. United Brotherhood of Carpenters and Joiners of America, Local 1669 v. Finn Way General Contractors Inc., 2011 CanLII 28357 (CRT Ont.).
} 
des salariés syndiqués, ils n'acceptent de réembaucher que ceux qui consentent à signer un document établissant qu'ils ont été forcés de prendre une carte syndicale ${ }^{39}$. Un employeur a même prétendu que tous les salariés, membres du syndicat, avaient démissionné, alors qu'ils avaient quitté le travail après avoir constaté que l'équipement fourni par l'employeur était inadéquat et tenté de l'aviser pour recevoir de nouvelles directives. Il a par la suite refusé les réembaucher afin qu'ils ne soient plus en poste au moment du dépôt de la requête en accréditation ${ }^{40}$.

La limitation des échanges entre le syndicat et les travailleurs, soit en lui refusant l'accès aux lieux physiques de travail, soit en bloquant les possibilités de communication entre le syndicat et ses membres (retrait des informations affichées sur le panneau syndical, interdiction de l'utilisation des équipements de l'entreprise, etc.) est présente dans 21 cas $^{41}$. Très souvent, les comportements de l'employeur et de ses représentants constituent des pratiques discriminatoires qui visent soit un individu, souvent le représentant syndical, soit le collectif salarié. Par exemple, un employeur a décidé d'affecter le représentant syndical dans un autre lieu de travail pour l'isoler, mieux le surveiller et diminuer sa capacité d'action ${ }^{42}$. Dans un autre cas, l'employeur a tenté de séparer les salariés et de les empêcher de communiquer les uns avec les autres avant la tenue d'un vote syndical. En ce qui concerne le blocage des moyens de communication, un commissaire ${ }^{43}$ a rappelé que « [1]es communications du syndicat avec ses membres constituent une assise fondamentale de l'activité associative », et qu' " un syndicat doit pouvoir bénéficier d'une grande liberté d'expression et être à l'abri des représailles de l'employeur » pour remplir ses fonctions, a fortiori en période de négociation collective ${ }^{44}$.

\section{À quel moment les stratégies anti-syndicales sont-elles les plus utilisées?}

Les tactiques anti-syndicales peuvent être déployées à différents moments de la vie professionnelle d'un salarié ou de l'organisation collective des salariés. Dans le cadre de notre étude, nous nous sommes intéressées au cycle d'accréditation d'un syndicat, que nous avons scindé en quatre grandes périodes : 1) avant le dépôt de la requête en accréditation, 2) entre le dépôt de la requête et l'obtention de l'accréditation, 3 ) entre l'accréditation et la conclusion d'une première convention collective et, finalement, 4) après la conclusion de la première convention collective. Cette division reflète

39. CAW Local 462 of National Automobile, Aerospace, Transportation and General Workers' Union (CAW-Canada) v. Lewis Bakeries (1966) Inc., 2003 CanLII 41019 (CRT Ont.).

40. United Brotherhood of Carpenters and Joiners of America, Local 1946 v. VanderWal Homes \& Commercial Group, 2011 CanLII 75806 (CRT Ont.).

41. Voir par exemple : Syndicat des travailleuses et travailleurs du Centre de santé et services sociaux de Québec-Sud (CSN) c. Centre de Santé et des services sociaux, 2012 QCCRT 0312 [cas CSSS Québec-Sud].

42. Voir cas Eagle Plumbing Contractors Inc., supra note 6.

43. C'est-à-dire un juge administratif de la commission des relations de travail.

44. Cas CSSS Québec-Sud, supra note 41, au paragraphe 31. 
les moments généralement considérés dans la littérature comme « charnières » pour l'implantation ou la survie d'une organisation syndicale.

En ce qui concerne spécifiquement les pratiques discriminatoires, un constat s'impose : les cas ne sont pas répartis de la même façon au Québec et en Ontario. Vingt-deux litiges ontariens sur 26 concernent ainsi la période avant l'obtention de l'accréditation, tandis que les quatre autres portent sur des événements ayant eu lieu après la conclusion de la convention. Au Québec en revanche, alors que 25 litiges sont fondés sur des faits survenus avant l'obtention de l'accréditation, la majorité des cas recensés ont lieu pendant la quatrième période (68 cas sur 101), c'est-à-dire après la conclusion d'une première convention. Si dans les cas ontariens les pratiques discriminatoires visent à empêcher l'implantation d'un syndicat dans une entreprise, dans les cas québécois elles se manifestent surtout une fois le syndicat accrédité. L'une des pistes d'explication de cette différence réside dans les pouvoirs qui sont conférés à la commission des relations du travail ontarienne, qui lui permettent de compenser les recours à des pratiques déloyales de travail par l'accréditation syndicale (WILLOWS, Schetagne, 2011), un pouvoir dont ne bénéficie pas la commission québécoise. Pour les syndicats ontariens, il y a donc un avantage à déposer une plainte très tôt dans le processus. Dans le cas du Québec, les syndicats peuvent décider de ne pas se plaindre à l'étape de l'accréditation syndicale car les délais qu'implique ce genre de procédure peuvent nuire à leur campagne. Ils peuvent alors préférer tenter de contourner les stratégies des employeurs et chercher à obtenir tout de même leur accréditation. Une autre explication tient peut-être à la procédure d'accréditation accélérée prévue au Québec qui, conjuguée au recours possible à l'arbitrage pour la conclusion d'une première convention collective, est susceptible de réduire les cas de pratiques déloyales dans les premières périodes.

Par ailleurs, quelques cas s'étendent sur plus d'une période (3 cas au Québec et 4 en Ontario), ce qui est peut-être l'indice de la présence de discrimination continue envers une association syndicale ou un militant. Notons cependant qu'une discrimination continue peut également se manifester à l'intérieur d'une seule et même période. Parmi les cas répertoriés, l'employeur modifie de manière significative les conditions de travail d'une salariée qui a discuté avec ses collègues de l'opportunité de se syndiquer (cf. Deshaies c. Académie internationale Zig Zag, 2010 QCCRT 0308). À la suite d'une campagne de syndicalisation, l'employeur décide de licencier cette salariée, ainsi que deux autres militants syndicaux après avoir constaté qu'ils font partie de l'équipe de négociation. Dans sa décision, la commission des relations de travail déclare que les changements apportés aux tâches de la plaignante constituent un licenciement déguisé. La commission annule l'évolution des conditions de travail et le licenciement qui s'ensuit, et ordonne la réintégration de la salariée à son poste. L'affaire Journeymen and Apprentices of the Plumbing and Pipefitting Industry of the United States and Canada v. Eagle Plumbing Contractors Inc. ${ }^{45}$ est comparable :

45. Cas Eagle Plumbing Contractors Inc., supra note 6. 
l'employeur déplace l'un de ses salariés, instigateur de la campagne de syndicalisation, sur un autre chantier où il est isolé de ses collègues de travail et davantage surveillé, et par conséquent moins susceptible de faire signer des cartes d'adhésion syndicale. Une fois le vote d'accréditation tenu, le travailleur voit ses responsabilités diminuer. De plus, on lui refuse l'augmentation de salaire dont bénéficient pourtant ses collègues.

Cette analyse des stratégies anti-syndicales et des pratiques de discrimination syndicale au sein de deux provinces canadiennes permet de dégager certains grands constats. Premièrement, elle montre la capacité stratégique qu'a l'acteur patronal d'élaborer des stratégies diverses qui lui permettent de maintenir un environnement de travail en phase avec son idéologie à l'égard de la syndicalisation (LAROCHE, 2012). Plus spécifiquement, notre analyse révèle que les employeurs québécois et ontariens ont utilisé une gamme étendue de tactiques pour combattre la syndicalisation dans les milieux de travail, et ce, à différents moments de la vie du syndicat. Nos observations montrent que les pratiques de discrimination syndicale utilisées sont variées et que si elles ont souvent visé un seul salarié, elles ont parfois concerné un groupe ou même l'ensemble du collectif de salariés. Dans certains cas, les indices importants que sont le nombre de tactiques anti-syndicales mobilisées par un même employeur nous ont permis d'apprécier l'intensité de l'opposition patronale à la syndicalisation. Si dans plusieurs cas, l'employeur ne met en œuvre qu'une ou deux tactiques anti-syndicales, d'autres ont fait preuve d'une opposition nettement plus vive. Dans un cas par exemple, l'employeur a recouru à 11 tactiques appartenant aux quatre types de campagnes que nous avons identifiées.

Deuxièmement, nos données révèlent une tendance comparable à ce que l'on observe dans le contexte états-unien, puisque les employeurs étudiés ont témoigné d'une nette préférence pour les stratégies dites « suppressives », les plus agressives (BRONFENBRENNER, 2009). Ces observations soulèvent différents types de questions. Pourquoi les travailleurs se plaignent moins de subir des tactiques associées aux stratégies dites de substitution, donc aux campagnes de cooptation ou de contrôle ? Est-ce parce que les travailleurs identifient plus difficilement ces pratiques comme étant anti-syndicales ? Notre méthode d'analyse ne permet pas de répondre à ces questions. Des recherches futures pourront le faire, par exemple en privilégiant une approche qualitative afin d'approfondir la compréhension d'un phénomène qui demeure complexe.

Enfin, la comparaison des situations ontarienne et québécoise met en évidence que dans ce dernier cas la protection législative n'est que de courte durée : les tactiques anti-syndicales, en particulier les pratiques discriminatoires, sont mises en œuvre une fois la première convention collective conclue. Dès lors, même une victoire syndicale lors de l'étape d'accréditation ne garantit pas le maintien d'une solidarité syndicale ou l'appui des travailleurs à leur organisation représentative, l'employeur étant clairement en mesure de déployer nombre de stratégies pour nuire au syndicat. À l'heure actuelle, 
et malgré la protection offerte par les différentes dispositions législatives analysées, les employeurs continuent de recourir, nous l'avons montré, à des pratiques antisyndicales et discriminatoires. Suivant Sara SLINN (2008), on peut considérer que les employeurs ont tendance à mobiliser des tactiques anti-syndicales ou discriminatoires s'ils considèrent comme non dissuasives les sanctions associées. Tel est visiblement le cas dans les deux provinces canadiennes que nous avons étudiées. Malgré les pouvoirs de réparation des commissions des relations du travail, les sanctions imposées en cas de plaintes sont manifestement jugées plus acceptables que la présence d'un syndicat dans leur organisation. Les travaux futurs devront chercher à identifier et expliquer les failles des systèmes québécois et ontarien qui ne protègent pas de manière effective le droit d'association des travailleurs.

\section{LÉGISLATION}

Code du travail du Québec, R.L.R.Q., c. C-27.

Loi de 1995 sur les relations de travail, L.O. 1995, c. 1, annexe A, telle qu'amendée par L.O. 2014, c. 10, annexe 3 .

\section{BIBLIOGRAPHIE}

Aidt T. S, Tzannatos Z. (2008), "Trade Unions, Collective Bargaining, and Macroeconomic Performance: A Review", Industrial Relations Journal, vol. 39, n 4, pp. 258-295.

Amossé T., Denis J.-M. (coordination et introduction) (2016), « La discrimination syndicale en question : la situation en France », Travail et Emploi, $\mathrm{n}^{\circ} 145$.

Bentham K. J. (2002), "Employer Resistance to Union Certification: A Study of Eight Canadian Jurisdictions”, Relations industrielles/Industrial Relations, vol. 57, n ${ }^{\circ}$ 1, pp. 159-187.

Béroud S., Denis J.-M., Desage G., Giraud B., Pélisse J. (2008), La lutte continue ? Les conflits du travail dans la France contemporaine, Bellecombe-en-Bauges, Éditions du Croquant.

BRONFENBRENNER K. (1994), "Employer Behavior in Certification Elections and First-Contract Campaigns: Implications for Labor Law Reform", in Friedman S. et al. (eds.), Restoring the Promise of American Labor Law, Ithaca (New York), ILR Press, pp. 75-89.

Bronfenbrenner K. (1996), "Final Report: The Effects of Plant Closing or Threats of Plant Closing on the Right of Workers to Organize", Key Workplace Documents, International Publications, Cornell University, ILR School.

BRONFENBRENNER K. (2009), "No Holds Barred: The Intensification of Employer Opposition to Organizing”, Briefing Paper, n $^{\circ} 235$, Washington D. C., Economic Policy Institute.

BoIvin J. (dir.) (2010), Introduction aux relations industrielles, $2^{\mathrm{e}}$ éd, Montréal, Gaëtan Morin Éditeur. 
CHAPPE V.-A (2013), « Dénoncer en justice les discriminations syndicales : contribution à une sociologie des appuis conventionnels de l'action judiciaire », Sociologie du travail, vol. 55, $\mathrm{n}^{\mathrm{o}} 3$, pp. 302-321.

ChAPPE V.-A (2015), « Les discriminations syndicales saisies par le droit à PSA », La Nouvelle Revue du travail [en ligne], $\mathrm{n}^{\circ} 7$; en ligne : http://nrt.revues.org/2324, consulté le 6 janvier 2017.

Clément H. (2013), « Petite phénoménologie du despotisme d'entreprise », Agone, nº 50, pp. 65-88.

Cooper R., Ellem B., Briggs C., Broek (VAn Den) D. (2009), "Anti-Unionism, Employer Strategy, and the Australian State, 1996-2005”, Labour Studies Journal, vol. 34, n 3, pp. 339-362.

Coutu M., Fontaine L. L., Marceau G., Coiquaud U. (2013), Droit des rapports collectifs du travail au Québec, $2^{e}$ édition, volume 1 : le régime général, Cowansville, Yvon Blais.

DebrégeAs A. (2013), « Entretien d'Anne Debrégeas, syndicaliste (SUD-Énergie). À propos de Répression et de discrimination syndicales », Agone, $\mathrm{n}^{\circ}$ 50, pp. 19-32.

Dundon T. (2002), "Employer Opposition and Union Avoidance in the UK", Industrial Relations Journal, vol. 33, n 3, pp. 234-245.

Dundon T., Cullinane N., Harney B. (2006), “The Ideology of Union Busting”, International Union Rights Journal, vol. 13, n ${ }^{\circ}$ 2, pp. 5-6.

Dundon T., Gollan P. J. (2007), "Re-conceptualizing Voice in the Non-Union Workplace", International Journal of Human Resource Management, vol. 18, n ${ }^{\circ}$ 7, pp. 1182-1198.

Dundon T., Harney B., Cullinane T. (2010), “De-Collectivism and Managerial Ideology: Towards an Understanding of Trade Union Opposition”, International Journal of Management Concepts and Philosophy, vol. 4, n ${ }^{\circ}$ 3-4, pp. 267-281.

EDWARDS T. (2004), “Corporate Governance, Industrial Relations and Trends in CompanyLevel Restructuring in Europe: Convergence Towards the Anglo-American Model?", Industrial Relations Journal, vol. 35, n 6, pp. 518-535.

FAirbrother P., Yates C. (2003), "The Revival of Industrial Unions in Canada: The Extension and Adaptation of Industrial Union Practices to the New Economy", in Fairbrother P., Yates C. (eds.), Trade Unions in Renewal: A Comparative Study, London, Continuum, pp. 221-243.

Freeman R. B., Kleiner M. M. (1990), "Employer Behavior in the Face of Union Organizing Drives", Industrial and Labor Relations Review, vol. 43, n 4, pp. 351-365.

Gall G. (2002), “Employer Opposition to Union Recognition”, in Gall G. (ed.), Union Organizing: Campaigning for Trade Union Recognition, London, Routledge, pp. 79-96.

Gall G. (2004), "British Employer Resistance to Trade Union Recognition”, Human Resource Management Journal, vol. 14, $\mathrm{n}^{\circ}$ 2, pp. 36-53.

Gall G., Dundon T. (eds.) (2013), Global Anti-Unionism: Nature, Dynamics, Trajectories and Outcomes, Basingstoke, Palgrave Macmillan. 
GIRAUD B. (2013), « Derrière la vitrine du "dialogue social” : les techniques managériales de domestication des conflits du travail », Agone, n ${ }^{\circ} 50$, pp. 33-64.

Hickey R., Kuruvilla S., Lakhani T. (2010), “No Panacea for Success: Member Activism, Organizing and Union Renewal”, British Journal of Industrial Relations, vol. 48, n 1, pp. 53-83.

Hurd R. W., Uehlein J. B. (1994), "Patterned Responses to Organizing: Case Studies of the Union-busting Convention", in Friedman S. et al. (eds.), Restoring the Promise of American Labor Law, Ithaca (New York), ILR Press, pp. 61-74.

JULLIARD É. (2013), « Les syndicats américains face aux stratégies managériales d'entrave du syndicalisme », Agone, n ${ }^{\circ}$ 50, pp. 89-114.

Juravich T., Bronfenbrenner K. (1998), "Preparing for the Worst: Organizing and Staying Organized in the Public Sector", in Bronfenbrenner K., Friedman S., Hurd R. W., Oswald R. A., Seeber R. L. (eds.), Organizing to Win: New Research on Union Strategies, Ithaca (New York), London, ILR Press, pp. 262-282.

Kumar P., Schenk C. (2005), "Union Renewal and Organizational Change: A Review of the Literature", in Kumar P., Schenk C. (eds.), Paths to Union Renewal: Canadian Experiences, Peterborough (ON), Broadview Press, pp. 29-60.

LAROCHE M. (2012), «Action collective patronale sur fond de crise : vers une reconfiguration du modèle associatif », Revue canadienne de sociologie, vol. 49, n 3, pp. 271-291.

LAROChe M., Bernier M.-È., Dupuis M. (2015), « Du caractère multidimensionnel des tactiques anti-syndicales : constats empiriques québécois », Canadian Labour \& Employment Law Journal, vol. 18, ${ }^{\circ}$ 2, pp. 557-594.

Martinello F., Yates C. (2004), "Union and Employer Tactics in Ontario Organising Campaigns", in Lewin D., Gollan P. (eds.), Advances in Industrial and Labor Relations, vol. 13, Bingley, Emerald, pp. 157-190.

PeEtz D. (2002), "Decollectivist Strategies in Oceania”, Relations industrielles/Industrial Relations, vol. 57, n 2, pp. 252-281.

Penissat É. (dir.) (2013), « Réprimer et domestiquer : stratégies patronales », Agone, $\mathrm{n}^{\circ} 50$.

PIER C. (2007), “Discounting Rights. Wal-Mart's Violation of US Workers' Right to Freedom of Association", Human Rights Watch, vol. 19, n 2 ; en ligne : http://www.hrw.org/sites/default/ files/reports/us0507webwcover.pdf, consulté le 6 janvier 2017.

Riddell C. (2001), "Union Suppression and Certification Success", Canadian Journal of Economics, vol. 34, n 2, pp. 396-410.

RiMBERT P., CRESPo S. (2004), « Devenir syndicaliste ouvrier. “Journal” d'un délégué CGT de la métallurgie », Actes de la recherche en sciences sociales, $\mathrm{n}^{\circ} 155$, pp. 34-75.

Rouillard J. (2004), Le Syndicalisme québécois. Deux siècles d'histoire, Montréal, Boréal.

Roy D. (1980), "Repression and Incorporation. Fear Stuff, Sweet Stuff and Evil Stuff: Management's Defences Against Unionization in the South", in Nichols T. (ed.), Capital and Labour: Studies in the Capitalist Labour Process, London, Athlone in association with Fontana, pp. 395-415. 
SuInN S. (2008), "No Right (to Organize) Without a Remedy: Evidence and Consequences of the Failure to Provide Compensatory Remedies for Unfair Labour Practices in British Columbia", McGill Law Journal/Revue de droit de McGill, vol. 53, nº 4, pp. 687-737.

SPIRE R. (2006), «Agir contre la discrimination syndicale au travail : le droit en pratique », Le Droit ouvrier, nouvelle série, $\mathrm{n}^{\circ}$ 693, pp. 170-218 et 222-230.

Supiot A. (dir.) (1999), Au-delà de l'emploi. Transformations du travail et devenir du droit du travail en Europe, Rapport pour la Commission des Communautés européennes, avec la collaboration de l'université Carlos III de Madrid, Paris, Flammarion.

Tanguay-Lavallée B., Houle A., Marotte J., Nadeau C., Poulin M. (2012), « Les différents régimes de représentation collective au Québec », Regards sur le travail [en ligne], vol. 9, numéro spécial - Forum 2012, pp. 1 - 19 ; en ligne : https://www.travail.gouv.qc.ca/fileadmin/ fichiers/Documents/regards_travail/vol09-forum/01_Regimes_de_representation_collective. pdf, consulté le 6 janvier 2017.

Thomason T. (1994), "The Effect of Accelerated Certification Procedures on Union Organizing Success in Ontario", Industrial and Labor Relations Review, vol. 47, n 2 2, pp. 207-226.

Thomason T., Pozzebon S. (1998), "Managerial Opposition to Union Certification in Quebec and Ontario", Relations industrielles/Industrial Relations, vol. 53, $\mathrm{n}^{\circ}$ 4, pp. 750-771.

Thompson M., Rose J. B., Smith A. (eds.) (2003), Beyond the National Divide: Regional Dimensions of Industrial Relations, Canadian Industrial Relations Association/Association canadienne des relations industrielles, School of Public Policies, Queen's University, Montréal et Kingston, McGill-Queen's University Press.

Trudeau G., VeIlleuX D. (1995), « Le monopole nord-américain de représentation syndicale à la croisée des chemins », Relations industrielles/Industrial Relations, vol. 50, n ${ }^{\circ}$ 1, pp. 9-38.

Turner L., Katz H. C., Hurd R. W. (2001), Rekindling the Movement: Labor's Quest for Relevance in the Twenty-First Century, Ithaca, London, ILR Press.

WiLlows J., Schetagne S. (2011), "Mapping Fundamental Labour Relations Laws in Canada -2011-2012”, Rapport de recherche, n ${ }^{\circ}$ 50, Ottawa, Canadian Labour Congress. 